\title{
ON BADLY APPROXIMABLE COMPLEX NUMBERS
}

\author{
R. ESDAHL-SCHOU \\ Department of Mathematical Sciences, Faculty of Science, University of Aarhus, Ny Munkegade, \\ Building 1530, DK-8000 Aarhus C, Denmark \\ e-mail:estel@imf.au.dk \\ and S. KRISTENSEN \\ Department of Mathematical Sciences, Faculty of Science, University of Aarhus, Ny Munkegade, \\ Building 1530,DK-8000 Aarhus C, Denmark \\ e-mail:sik@imf.au.dk
}

(Received 11 March 2009; accepted 26 October 2009)

\begin{abstract}
We show that the set of complex numbers which are badly approximable by ratios of elements of the ring of integers in $\mathbb{Q}(\sqrt{-D})$, where $D \in\{1,2,3,7,11,19$, $43,67,163\}$ has maximal Hausdorff dimension. In addition, the intersection of these sets is shown to have maximal dimension. The results remain true when the sets in question are intersected with a suitably regular fractal set.
\end{abstract}

2000 Mathematics Subject Classification. 11J83.

1. Introduction. Diophantine approximation over the complex numbers has been studied in a number of papers. In the present paper, we are concerned with the approximation of complex numbers by ratios of elements in the ring of integers in $\mathbb{Q}(\sqrt{-D})$, whenever this ring has unique factorisation. We denote these rings by $\mathcal{O}_{D}$. The question of unique factorisation in these rings goes back to Gauss. It was eventually shown by Stark [11] that $\mathcal{O}_{D}$ has unique factorisation if and only if $D \in\{1,2,3,7,11,19,43,67,163\}$, the values listed in the abstract. An alternative proof using linear forms in logarithms was obtained by Baker [1].

Let $D \in\{1,2,3,7,11,19,43,67,163\}$ and consider initially the inequality

$$
\left|z-\frac{p}{q}\right|<\frac{2 D^{3 / 2}}{|q|^{2}}
$$

where $z \in \mathbb{C}$ and $p, q \in \mathcal{O}_{D}, q \neq 0$. It follows from Minkowski's linear forms theorem that for any $z \in \mathbb{C}$, equation (1) has infinitely many solutions $p, q \in \mathcal{O}_{D}$. A proof of this can be found in [4] in the case $D=1$. We leave it to the reader to extend this to other values of $D$.

No doubt, the constant $2 D^{3 / 2}$ in equation (1) can be improved, as we make very coarse estimates in the proof that there are infinitely many solutions. However, it cannot be made arbitrarily small, as we will show in this paper. We consider the converse inequality,

$$
\left|z-\frac{p}{q}\right| \geq \frac{K(z)}{|q|^{2}} \text { for all } p, q \in \mathcal{O}_{D}, q \neq 0 .
$$


We will call the set of complex numbers $z \in \mathbb{C}$ for which equation (2) is satisfied the set of badly approximable complex numbers with respect to $\mathcal{O}_{D}$, denoted $\mathbf{B a d}_{D}$.

We require that $\mathcal{O}_{D}$ has unique factorisation in order to make the right-hand sides of equation (1) and equation (2) independent of the representative of $p / q$ on the left-hand side. If unique factorisation fails, we could have $p / q=p^{\prime} / q^{\prime}$, both on lowest terms, but with $|q| \neq\left|q^{\prime}\right|$, which would cause some ambiguity. Additionally, we will use unique factorisation in the course of the proof.

A. Schmidt [10] studied analogues of continued fractions in the complex numbers. Among other things, he proved that $\operatorname{Bad}_{1} \neq \varnothing$, and obtained quantitative information on the set of best possible values for $K(z)$ as $z$ varies. When the set of complex numbers is considered as the limit set of the Picard group, it can be derived from results of Bishop and Jones [3] or Fernandez and Melián [6] that the set $\mathbf{B a d}_{1}$ has maximal Hausdorff dimension. This is also proved in [4] by more elementary means, using the framework of Schmidt games (see below). In [8] it is shown that in addition $\operatorname{Bad}_{1} \cap K$ is of maximal dimension whenever $K$ supports a measure satisfying several technical conditions. An example of such a set $K$ is the Sierpínski gasket. We will return to these technical conditions below.

The objective of the present paper is to extend the result of [8] to any $D \in$ $\{1,2,3,7,11,19,43,67,163\}$, using the methods of [4]. A desirable property of that method is the dimension result that is stable under finite intersection. Specifically, we prove the following theorem.

THEOREM 1. Let $K \subseteq \mathbb{C}$ be a compact set supporting a measure $\mu$ for which there exist constants $a, b, \delta, r_{0}>0$ such that for any $z \in K$ and any $r \leq r_{0}$,

$$
a r^{\delta} \leq \mu(B(z, r)) \leq b r^{\delta}
$$

Then, for any subset $E \subseteq\{1,2,3,7,11,19,43,67,163\}$,

$$
\operatorname{dim}_{H} K \cap \bigcap_{D \in E} \operatorname{Bad}_{D}=\operatorname{dim}_{H} K=\delta
$$

REMARKS.

(i) The condition on $K$ is evidently satisfied for any closed ball in $\mathbb{C}$ with $\mu$ being the two-dimensional Lebesgue measure. In addition, it was shown by Hutchinson [7] that the condition is satisfied for the attractor of a family of similarities satisfying the open set condition. Classical examples are the Sierpínski gasket, the von Koch curve and the Cantor middle third set.

(ii) The condition on the measure of a ball decaying as a power of the radius of the ball is slightly stronger than the technical conditions in [8]. It is likely that the assumption can be weakened to those of [8], but at the cost of additional technicalities. We have chosen to keep the statements and results as simple as possible.

(iii) In fact we will prove rather more than maximal dimension. Indeed, we will show that the sets $\mathbf{B a d}_{D}$ are winning for a Schmidt game (see below), which is a stronger statement.

(iv) It is possible to deduce Theorem 1 in the case $\# S=1$ from the general framework of [8]. With additional work, it may well be possible to obtain the full theorem from this set-up. However, that framework will not prove that 
the set is winning. It would also be considerably less elementary and not as self-contained as the present proof.

2. Proof of Theorem 1. In order to prove Theorem 1, we will use the Schmidt game introduced by W. M. Schmidt in [9]. Let $K$ be a set as in the statement of the theorem, let $S \subseteq K$ and let $\alpha, \beta \in(0,1)$. The $(\alpha, \beta, K ; S)$-game is played by two players Black and White as follows: Initially, Black chooses a ball $B_{1} \subseteq \mathbb{C}$ with centre $x_{1} \in K$ and radius $\rho=\rho\left(B_{1}\right)$ for some $\rho>0$. White then chooses a ball $W_{1} \subseteq B_{1}$ with centre $y_{1} \in K$ and radius $\rho\left(W_{1}\right)=\alpha \rho\left(B_{1}\right)$. Now Black chooses a ball $B_{2} \subseteq W_{1}$ with centre $x_{2} \in K$ and radius $\rho\left(B_{2}\right)=\beta \rho\left(W_{1}\right)$ and so on ad infinitum. The outcome of the game is the unique point $x$ such that $\bigcap_{n=1}^{\infty} B_{n}=\{x\}$. Since $K$ is compact, this is an element in $K$. If in addition $x \in S$, White wins the game. Otherwise Black wins.

In Schmidt's paper [9], the game was played in an arbitrary metric space. Here, we use a sub-space $K$ of the ambient metric space $\mathbb{C}$, and require that the centres of the balls lie in the sub-space. We are going to apply the set-up to study numbers which are badly approximated by distinguished elements in the ambient space $\mathbb{C}$. To make the distinction clear, we have opted to put the compact set $K$ into the definition of the game.

We will call a set $S(\alpha, \beta, K)$-winning if White can always win the $(\alpha, \beta, K ; S)$-game. If for some $\alpha \in(0,1)$, the set $S$ is $(\alpha, \beta, K)$-winning for all $\beta \in(0,1)$, we will say that $S$ is $(\alpha, K)$-winning. In order to prove Theorem 1 , we will need three Lemmas.

LEMMA 2. Let $\left\{S_{i}\right\}$ be a sequence of subsets of $K$, all of which are $(\alpha, K)$-winning for some $\alpha>0$. Then $\bigcap_{i=1}^{\infty} S_{i}$ is $(\alpha, K)$-winning.

Lemma 3. Let $S \subseteq K$ and suppose that $S$ is $(\alpha, K)$-winning for some $\alpha>0$. Then

$$
\operatorname{dim}_{H} S=\operatorname{dim}_{H} K
$$

Lemma 4. Let $D \in\{1,2,3,7,11,19,43,67,163\}$ and $K$ be as in the statement of Theorem 1. Then $\operatorname{Bad}_{D} \cap K$ is $(\alpha, K)$-winning for any $\alpha \leq \frac{r_{0}}{24\left(1+r_{0}\right)}\left(\frac{a^{2}}{2 b^{2}}\right)^{1 / \delta}$.

Proof of Theorem 1 assuming Lemmas 2-4. Let $\alpha \leq \frac{r_{0}}{24\left(1+r_{0}\right)}\left(\frac{a^{2}}{2 b^{2}}\right)^{1 / \delta}$, so that $\operatorname{Bad}_{D} \cap$ $K$ is $(\alpha, K)$-winning. It follows that $\bigcap_{D \in E} \operatorname{Bad}_{D} \cap K$ is $(\alpha, K)$-winning by Lemma 2 . The theorem now follows immediately from Lemma 3.

It remains to prove the lemmas. Lemma 2 is just [9, Theorem 2]. In order to prove Lemma 3, we invoke Corollary 1 of [9, Theorem 6]. Adapted to our set-up, this result is stated as follows.

Lemma 5 (Corollary 1 of $[9$, Theorem 6]). Let $N(\beta) \in \mathbb{N}$ be such that any ball $B$ with centre in $K$ and radius $\rho$ contains $N(\beta)$ balls with centres in $K$ of radius $\beta \rho$ with pairwise disjoint interiors. Then, for any $(\alpha, \beta, K)$-winning set $S$,

$$
\operatorname{dim}_{H} S \geq \frac{\log N(\beta)}{|\log (\alpha \beta)|} .
$$

Some remarks are in order about the proof of Lemma 5. In [9], the result is stated only for $K$ being a Hilbert space. Evidently, this is not necessarily the case for $K$. 
However, the only place in the proof of the Lemma in [9], where the Hilbert space property is needed is in Lemma 20, where an upper estimate on the number of disjoint balls intersecting a fixed ball is obtained. Evidently, $K$ inherits this property from $\mathbb{C}$, which is of course a Hilbert space.

In addition to Lemma 5, we will need an elementary covering result. The result we need states that if $\left\{B_{i}\right\}$ is a finite collection of balls in a compact metric space of equal radius, then there is a disjoint sub-collection $\left\{B_{i_{j}}\right\}$ such that

$$
\bigcup_{i} B_{i} \subseteq \bigcup_{j} 3 B_{i_{j}} .
$$

A proof of this fact can be found in [2, Lemma 4].

Proof of Lemma 3. From the conditions on $K$, it follows that $\operatorname{dim}_{H} K=\delta$, so we will prove that the Hausdorff dimension of any $(\alpha, K)$-winning set is at least equal to $\delta$.

Let $\beta \in(0,1 / 3)$ be arbitrary, but fixed. By Lemma 5, we need only estimate $N(\beta)$ to obtain a lower estimate on the dimension. Let $B$ be a ball with centre in $K$ and radius $\rho \leq r_{0}$, where $r_{0}$ is the constant from the statement of Theorem 1 , so that

$$
\mu\left(\frac{1}{2} B\right) \geq a 2^{-\delta} \rho^{\delta} .
$$

Take a covering of $\frac{1}{2} B \cap K$ with balls centred in $K$ with radius $\beta \rho$. By equation (4) applied to the compact metric space $K$, we can refine this to a disjoint collection $B_{1}, \ldots, B_{k}$, such that the full collection, and hence $\frac{1}{2} B \cap K$, is covered by $3 B_{1}, \ldots, 3 B_{k}$. Evidently, the $B_{i}$ are all contained in $B$, pairwise disjoint and centred in $K$. It remains to estimate $k$.

Since

$$
\mu\left(B_{i}\right) \leq b 3^{\delta} \rho^{\delta}, \quad 1 \leq i \leq k,
$$

we find that

$$
2^{-\delta} a \rho^{\delta} \leq k b 3^{\delta} \beta^{\delta} \rho^{\delta}
$$

so that

$$
k \geq \frac{a}{b 6^{\delta}} \beta^{-\delta}
$$

Hence, we have $N(\beta) \geq \frac{a}{b 6^{\delta}} \beta^{-\delta}$. Lemma 3 now follows on letting $\beta \rightarrow 0$ in equation (3).

In order to complete the proof of Theorem 1, it remains only to prove Lemma 4.

Proof of Lemma 4. Let $\alpha$ be as in the statement of the lemma, and let $\beta \in(0,1)$ be arbitrary. We proceed with the construction of a winning strategy for White.

Assume that the initial ball $B_{1}$ has radius $r_{1} \leq r_{0} / 2$, let $\epsilon=\alpha$ and let $R=\sqrt{\alpha \beta}$. We will prove that White has a strategy such that if $p, q \in \mathcal{O}_{D}$ satisfy

$$
(p, q)=1 \text { and } 0<|q|<R^{n}
$$


then for any $z \in B_{n+1}$,

$$
\left|z-\frac{p}{q}\right|>\frac{\epsilon}{|q|^{2}}
$$

We will accomplish this by induction in $n$.

For $n=0$, notice that the set of $q \in \mathcal{O}_{D}$ satisfying equation (5) is empty, and hence equation (6) is trivially satisfied. Hence, we suppose that $n>0$ and proceed by induction. We have already avoided counter-examples to equation (6) earlier in the game, and hence we need only worry about $q \in \mathcal{O}_{D}$ for which

$$
R^{n-1} \leq|q|<R^{n}
$$

We claim that in the next move, White need only worry about a single point $p / q$, subject to equation (7). Indeed, suppose that there are $p, p^{\prime}, q, q^{\prime} \in \mathcal{O}_{D}$ with $q$ and $q^{\prime}$ satisfying equation (7) as well as points $z, z^{\prime} \in B_{n}$ such that

$$
\left|z-\frac{p}{q}\right| \leq \frac{\epsilon}{|q|^{2}} \text { and }\left|z^{\prime}-\frac{p^{\prime}}{q^{\prime}}\right| \leq \frac{\epsilon}{\left|q^{\prime}\right|^{2}} .
$$

Then, since $\rho\left(B_{n}\right)=r_{1}(\alpha \beta)^{n-1}$,

$$
\left|\frac{p}{q}-\frac{p^{\prime}}{q^{\prime}}\right| \leq\left|z-\frac{p}{q}\right|+\left|z^{\prime}-\frac{p^{\prime}}{q^{\prime}}\right|+\left|z-z^{\prime}\right| \leq \frac{\epsilon}{|q|^{2}}+\frac{\epsilon}{\left|q^{\prime}\right|^{2}}+r_{1}(\alpha \beta)^{n-1} \leq R^{-2 n},
$$

by choice of parameters. On the other hand, if $p / q \neq p^{\prime} / q^{\prime}$, by unique factorisation,

$$
\left|\frac{p}{q}-\frac{p^{\prime}}{q^{\prime}}\right| \geq \frac{1}{\left|q q^{\prime}\right|}>R^{-2 n}
$$

a contradiction. Hence, $p / q=p^{\prime} / q^{\prime}$.

Suppose that there is a point $p / q$ to be avoided by White. Let $C$ be the open ball centred at $p / q$ of radius $\frac{\epsilon}{|q|^{2}}$. We will assume that $C \cap B_{n} \neq \emptyset$. As in the proof of Lemma $3, B_{n}$ contains disjoint balls $B_{1}, \ldots, B_{k}$ of radius $\alpha r_{n}$, where

$$
k \geq \frac{a}{b 6^{\delta}} \alpha^{-\delta} .
$$

We claim that under the assumptions, one of these is disjoint from $C$. Indeed, in order for one of the $B_{i}$ constructed above to have non-empty intersection with $C, B_{i}$ must be fully contained in $\left(1+2 \alpha r_{n}\right) C$. We estimate the number of balls having this property.

Evidently, the measure of an arbitrary ball $B \subseteq \mathbb{C}$ with radius $r \leq r_{0} / 2$ satisfies $\mu(B) \leq b 2^{\delta} r^{\delta}$. Hence, we estimate the measure of the ball $\left(1+2 \alpha r_{n}\right) C$ from above by

$$
\begin{aligned}
\mu\left(\left(1+2 \alpha r_{n}\right) C\right) & \leq b 2^{\delta}\left(\epsilon R^{-2(n-1)}+2 \alpha r_{1}(\alpha \beta)^{n-1}\right)^{\delta} \\
& =b\left(2 \epsilon+4 \alpha r_{1}\right)^{\delta}(\alpha \beta)^{(n-1) \delta} .
\end{aligned}
$$

On the other hand, for any $i \in\{1, \ldots, k\}$,

$$
\mu\left(B_{i}\right) \geq a\left(\alpha r_{n}\right)^{\delta}=a\left(\alpha r_{1}(\alpha \beta)^{n-1}\right)^{\delta} .
$$


Hence, the maximal number $k^{\prime}$ of disjoint $B_{i}$ that can be contained in $\left(1+2 \alpha r_{n}\right) C$ is bounded above by

$$
k^{\prime} \leq \frac{b\left(2 \epsilon+4 \alpha r_{1}\right)^{\delta}(\alpha \beta)^{(n-1) \delta}}{a\left(\alpha r_{1}(\alpha \beta)^{n-1}\right)^{\delta}}=\frac{b}{a}\left(\frac{2 \epsilon}{\alpha r_{1}}+4\right)^{\delta} \leq \frac{4^{\delta} b}{a}\left(\frac{1}{r_{0}}+1\right)^{\delta},
$$

by choice of $\epsilon$.

Now, by assumption on $\alpha$,

$$
k \geq 2 k^{\prime} \geq k^{\prime}+1
$$

In other words, White can choose a ball completely disjoint from $C$ in the next move. This completes the construction of the winning strategy, and hence the proof that $\mathbf{B a d}_{D}$ is $(\alpha, K)$-winning.

3. Concluding remarks. We have made no effort to find an optimal lower bound on the $\alpha$ for which the sets $\operatorname{Bad}_{D}$ are $(\alpha, K)$-winning. Evidently, by optimising the condition on $\alpha$, we immediately obtain such a bound depending on the measure theoretic structure of $K$. However the bounds obtained in this way are not optimal. In the case $K=\mathbb{C}$ and $D=1$, it is shown in [4] that $\operatorname{Bad}_{1}$ is $(\alpha, K)$-winning for any $\alpha<1 / 2$. The bound in Lemma 4 only shows that the set is winning for $\alpha<1 /(24 \sqrt{2})$.

An additional improvement on the bound on $\alpha$ could possibly be obtained by constructing White's strategy over more than one step. This was originally done by Schmidt in [9], where a strategy was devised to avoid the problematic fraction in $t$ steps, where $t$ is a positive integer satisfying $\alpha \beta \gamma \leq 2(\alpha \beta)^{t}<\gamma$. Again, we have made no such effort, and leave the problem to the interested reader. Note, however, that in general one cannot expect to reach the maximum possible value of $1 / 2$, as was observed by Fishman in the real case [5].

ACKNOWLedgements. Kristensen's research is funded by the Carlsberg Foundation.

\section{REFERENCES}

1. A. Baker, Linear forms in the logarithms of algebraic numbers. I, II, III, Mathematika 13 (1966), 204-216; 14 (1967), 102-107; 220-228.

2. V. Beresnevich, D. Dickinson and S. Velani, Measure theoretic laws for lim sup sets, Mem. Amer. Math. Soc. 179(846) (2006), x+91.

3. C. J. Bishop and P. W. Jones, Hausdorff dimension and Kleinian groups, Acta Math. 179(1) (1997), 1-39.

4. M. M. Dodson and S. Kristensen, Hausdorff dimension and Diophantine approximation, Fractal geometry and applications: A jubilee of Benoît Mandelbrot. Part 1, Proc. Symp. Pure Math. 72 (American Mathematical Society, Providence, RI, 2004) pp. 305-347.

5. L. Fishman, Schmidt's game, badly approximable matrices and fractals, J. Number Theory 129(9) (2009), 2133-2153.

6. J. L. Fernández and M. V. Melián, Bounded geodesics of Riemann surfaces and hyperbolic manifolds, Trans. Amer. Math. Soc. 347(9) (1995), 3533-3549. $713-747$

7. J. E. Hutchinson, Fractals and self-similarity, Indiana Univ. Math. J. 30(5) (1981), 
8. S. Kristensen, R. Thorn and S. Velani, Diophantine approximation and badly approximable sets, Adv. Math. 203(1) (2006), 132-169.

9. W. M. Schmidt, On badly approximable numbers and certain games, Trans. Amer. Math. Soc. 123 (1966), 178-199. $1-85$.

10. A. L. Schmidt, Diophantine approximation of complex numbers, Acta Math. 134(1975),

11. H. M. Stark, A complete determination of the complex quadratic fields of class-number one, Michigan Math. J. 14 (1967), 1-27. 